University of Nebraska - Lincoln

DigitalCommons@University of Nebraska - Lincoln

Center for Brain, Biology and Behavior: Papers \&

Publications

Brain, Biology and Behavior, Center for

4-9-2009

Frequency and spatial characteristics of highfrequency

neuromagnetic signals in childhood epilepsy

Jing Xiang

Yang Liu

Yingying Wang

Elijah G. Kirtman

Cincinnati Children's Hospital Medical Center Kotecha

See next page for additional authors

Follow this and additional works at: https://digitalcommons.unl.edu/cbbbpapers

Part of the Behavior and Behavior Mechanisms Commons, Nervous System Commons, Other Analytical, Diagnostic and Therapeutic Techniques and Equipment Commons, Other Neuroscience and Neurobiology Commons, Other Psychiatry and Psychology Commons, Rehabilitation and Therapy Commons, and the Sports Sciences Commons

This Article is brought to you for free and open access by the Brain, Biology and Behavior, Center for at DigitalCommons@University of Nebraska - Lincoln. It has been accepted for inclusion in Center for Brain, Biology and Behavior: Papers \& Publications by an authorized administrator of DigitalCommons@University of Nebraska - Lincoln. 


\section{Authors}

Jing Xiang, Yang Liu, Yingying Wang, Elijah G. Kirtman, Cincinnati Children's Hospital Medical Center Kotecha, Yangmei Chen, Xiaolin Huo, Hisako Fujiwara, Nat Hemasilpin, Ki Lee, Francesco T. Mangano, James Leach, Blaise Jones, Ton DeGrauw, and Douglas Rose 


\title{
Frequency and spatial characteristics of high- frequency neuromagnetic signals in childhood epilepsy
}

\author{
Jing Xiang ${ }^{1,2}$, Yang Liu ${ }^{1}$, Yingying Wang ${ }^{1}$, Elijah G. Kirtman ${ }^{1}$, \\ Rupesh Kotecha ${ }^{1}$, Yangmei Chen ${ }^{1}$, Xiaolin Huo ${ }^{1}$, \\ Hisako Fujiwara ${ }^{1,2}$, Nat Hemasilpin ${ }^{1,3}$, Ki Lee ${ }^{2}$, \\ Francesco T. Mangano ${ }^{3}$, James Leach ${ }^{4}$, Blaise Jones ${ }^{4}$, \\ Ton DeGrauw ${ }^{2}$, Douglas Rose ${ }^{1,2}$ \\ ${ }^{1}$ MEG Center \\ ${ }^{2}$ Division of Neurology \\ ${ }^{3}$ Division of Neurosurgery \\ ${ }^{4}$ Department of Radiology, Cincinnati Children's Hospital Medical Center, OH, USA \\ Received August 11, 2008; Accepted April 9, 2009
}

\begin{abstract}
Purpose. Invasive intracranial recordings have suggested that high-frequency oscillation is involved in epileptogenesis and is highly localized to epileptogenic zones. The aim of the present study is to characterize the frequency and spatial patterns of high-frequency brain signals in childhood epilepsy using a non-invasive technology. Methods. Thirty children with clinically diagnosed epilepsy were studied using a whole head magnetoencephalography (MEG) system. MEG data were digitized at $4000 \mathrm{~Hz}$. The frequency and spatial characteristics of high-frequency neuromagnetic signals were analyzed using continuous wavelet transform and beamformer. Threedimensional magnetic resonance imaging (MRI) was obtained for each patient to localize magnetic sources. Results. Twenty-six patients showed highfrequency $(100-1000 \mathrm{~Hz})$ components $(26 / 30,86 \%)$. Nineteen patients showed more than one high-frequency component (19/30, 63\%). The frequency range of high-frequency components varied across patients. The highest frequency band was identified around $910 \mathrm{~Hz}$. The loci of high-frequency epileptic activities were concordant with the lesions identified by magnetic resonance imaging for 21 patients (21/30, 70\%). The MEG source localizations of high-frequency components were found to be concordant with intracranial recordings for nine of the eleven patients who underwent epilepsy surgery $(9 / 11,82 \%)$. Conclusion. The results have demonstrated that childhood epilepsy was associated with high-frequency epileptic activity in a wide frequency range. The concordance of MEG source localization, MRI and intracranial recordings suggests that measurement of high-frequency neuromagnetic signals might provide a novel approach for clinical management of childhood epilepsy.
\end{abstract}

Key words: epilepsy, magnetoencephalography, high-frequency oscillation, children, beamformer, wavelet 
Detection of epileptic spikes has been used for diagnosis of epilepsy for several decades (Iwasaki et al. 2005, Ramantani et al. 2006). The conventional epileptic spike is typically defined as being 14-70 milliseconds $(14-70 \mathrm{~Hz})$ in duration. This is reflected by the commonly used low-pass filtering of the electroencephalography (EEG) signal around $70 \mathrm{~Hz}$ and the concomitant $200 \mathrm{~Hz}$ sampling rate (Firpi et al. 2007, Ramantani et al. 2006). Brain signals that have been clinically utilized in the past are limited to this low frequency range $(<100 \mathrm{~Hz})$. Recent reports have demonstrated that the brain generates highfrequency signals up to $1500 \mathrm{~Hz}$ (Okada et al. 2005). High-frequency brain signals (HFBS, $>70 \mathrm{~Hz}$ ) are also called high frequency oscillation (HFO), ripple or fast ripple. Intracranial recordings from clinical patients have shown that the epileptic brain is associated with abnormal high frequency signals (Bragin et al. 2002, Draguhn et al. 2000, Ylinen et al. 1995). Furthermore, the presence of high-frequency signals at $200-500 \mathrm{~Hz}$ has been confirmed in rodent epilepsy models but has not been observed in non-epileptic rodents, suggesting that certain HFBS are specifically associated with epileptogenesis (Firpi et al. 2007).

HFBS have also been found in functional brain activities such as somatosensory evoked magnetic fields (Okada et al. 2005). Thus, HFBS can be divided into two categories: physiogenic HFBS and pathogenic HFBS. The differences between physiogenic HFBS and pathogenic HFBS (e.g. epileptic HFBS) remains unclear (Le Van et al. 2006). One possible difference is related to frequency. As reported in human studies of seizure onset activity, epileptic HFBS mostly occur in the high gammafrequency band $(60-120 \mathrm{~Hz})$. Another class of HFBS is fast ripples; these are recorded in limited areas of epileptogenic limbic structures and in the $250-500 \mathrm{~Hz}$ range. A recent report has also showed that some physiological cognitive oscillations extend in the $50-200 \mathrm{~Hz}$ range (Jung et al. 2008). The highest frequency component has been found in the somatosensory system, which can reach up to $1500 \mathrm{~Hz}$ (Okada et al. 2005). Noticeably, the frequency range in the previous reports is very wide. Since the exact frequency bands of high-frequency epileptic activity and physiologic activity have not been reported, it is difficult to determine cut-off frequencies between physiogenic and pathogenic activities. In other words, a clear-cut separation between physiological and pathological activity is not well established.

Invasive intracranial recording is currently the "gold standard" for pre-operative evaluation of epilepsy surgery (Jirsch et al. 2006, Worrell et al. 2008). Thus, highfrequency epileptic signals in the human epileptic brain are identified mainly by clinical intracranial recordings (Bragin et al. 1999b, Bragin et al. 2002, Otsubo et al. 2008, Urrestarazu et al. 2006, Worrell et al. 2008). It has been noted that intracranial recording can cover only limited brain areas (usually the seizure onset zone).
More importantly, the invasive nature of intracranial recording limits its applications in the measurement of physiogenic HFBS. Thus, the development of noninvasive methods for precisely detecting HFBS is of paramount importance. The available techniques for noninvasively recording HFBS are EEG and MEG. Other functional neuroimaging modalities, such as functional magnetic resonance imaging (fMRI) and positron emission tomography (PET), do not have enough temporal resolution to capture high-frequency signals (Le Van et al. 2006, Raichle et al. 2001, Raichle and Snyder 2007, Salvador et al. 2008). In comparison to conventional EEG, MEG has advantages of spatial localization (Barkley and Baumgartner 2003). Recent reports have also shown that MEG has a potentially significant advantage for analysis of coherence of brain activity in various regions (Srinivasan et al. 2007). The recent development of magnetic signal processing technologies have made it possible to volumetrically estimate high-frequency neuromagnetic signals from the human brain (Dalal et al. 2008, Xiang et al. 2004), suggesting that MEG is a potentially useful tool for the study of high-frequency epileptic activity.

One main challenge in the analysis of HFBS by MEG or EEG is that high-frequency oscillations are obscured by lower frequency signals that may be much stronger than high-frequency signals (Jirsch et al. 2006, Worrell et al. 2008). In fact, even when occurring as a sustained discharge at the seizure onset, the HFBS is not always clearly apparent (Jirsch et al. 2006). Thus, it is necessary to use more advanced signal processing methods to identify HFBS. Several methods including Fast Fourier transformation and band-pass filtering (Bragin et al. 2002, Jacobs et al. 2008, Jirsch et al. 2006, Worrell et al. 2008) have been used for analysis of high-frequency activities such as fast ripple (200-500 Hz). A recent report (Xiang et al. 2004) has shown that accumulated spectrograms, a new method based on continuous wavelet transform, can detect highfrequency signals in a wide frequency band. Furthermore, virtual sensor techniques, a new method based on beamformer, can reveal morphological characteristics, location and distribution of epileptic activity similar to those shown by subdural EEG recordings (Oishi et al. 2006). The combination of accumulated spectrogram and virtual sensor technique has the potential to significantly increase the signal-to-noise ratio in high-frequency signals by removing signals in random frequency and space while maintaining frequency- and space-locked signals.

Taken together, intracranial recordings have confirmed that the epileptic brain generates high-frequency signals and the development of MEG and signal processing methods have shown the potential to capture high-frequency signals non-invasively. The purpose of the present study is to investigate high-frequency neuromagnetic activity in childhood epilepsy using a high-sampling rate MEG system and the latest signal processing methods including accumulated spectrogram and virtual sensor techniques. 
To our knowledge, high-frequency epileptic activity $(>100 \mathrm{~Hz}$ ) has rarely been studied with MEG in childhood epilepsy. To carefully exclude magnetic noise, the first part of this study was designed to analyze highfrequency neuromagnetic signals recorded with MEG in sensor space. The second part of this study was designed to analyze high frequency signals computed with virtual sensor techniques that may minimize magnetic noise such as power-line noise in source space. We hypothesize that epileptic high-frequency neuromagnetic signals can be non-invasively detected and localized by MEG with advanced signal processing algorithms. In comparison to existing invasive recording, non-invasive detection of high-frequency epileptic abnormality does not pose a risk to children. It can be used for all cooperative children with seizure(s) and may provide a novel technique for clinical management of epilepsy.

\section{Methods and materials}

\section{Patient selection}

Thirty clinical patients (age: 6-17 years, mean age 10; 14 female and 16 male) affected by localization-related epilepsy were retrospectively studied. Of the 30 patients: 15 patients had tuberous sclerosis, four patients had cortical dysplasia, four patients had tumor, two patients had dysembryoplastic neuroepithelial tumor (DNET), two patients had neonatal strokes, one patient had cavernous angioma and two patients had seizures after resection. All the selected patients had at least one visible lesion using structural images. Twelve of the patients underwent clinical intracranial recordings and eleven of the patients had epilepsy surgery. Furthermore, we had the following additional patient inclusion criteria: (1) head movement during MEG recording less than $5 \mathrm{~mm}$, (2) the deflections of all MEG data within 6 pT (the MEG data were considered "clean") and (3) epilepsy diagnosed clinically and magnetic resonance imaging (MRI) successfully obtained. All patients with epilepsy who underwent MEG studies in our hospital qualified for this study according to the criteria. This study was approved by the Institutional Review Board (IRB) at our hospital.

\section{MEG recording}

The MEG recordings were obtained in a magnetically shielded room (MSR) using a whole head CTF 275Channel MEG system (VSM MedTech Systems Inc., Coquitlam, BC, Canada) in the MEG Center at CCHMC. Before data acquisition commenced, a small coil was attached to the nasion, left and right pre-auricular points of each subject. These three coils were subsequently activated at different frequencies for measuring subjects' head positions relative to the MEG sensors. The system allowed head localization to an accuracy of $1 \mathrm{~mm}$. The sampling rate of the MEG recordings for patients was $4000 \mathrm{~Hz}$. All MEG data were recorded with a noise cancellation of third order gradients. The limitation of head movement during MEG recording was $5 \mathrm{~mm}$. Two minutes of spontaneous MEG data were recorded as one epoch; at least two epochs (total time was four minutes) were recorded for each patient. Patients were deprived of sleep the night before MEG and MR imaging studies. To identify system and environmental noise, we routinely recorded one MEG dataset without patient just before the experiment.

\section{MRI scan}

Three-dimensional Magnetization-Prepared Rapid Acquisition Gradient Echo (MP-RAGE) sequences were obtained for all subjects with a 3T scanner (Siemens Medical Solutions, Malvern, PA). MRI scans were performed by MRI technologists. Three fiduciary points were placed in identical locations to the positions of the three coils used in the MEG recordings to allow for an accurate co-registration of the two data sets. Subsequently, all anatomical landmarks digitized in the MEG study were made identifiable in the MR images.

\section{Intracranial EEG video monitoring}

Clinical intracranial electrocorticography $(\mathrm{ECoG})$ data were retrospectively analyzed with the MEG results. Of the 30 patients, 12 patients reported here had implantation of subdural electrodes and CCTV/EEG monitoring according to standard protocol at our hospital. Of these 12 patients, 11 patients had epilepsy surgery. Digital photos were taken before and during the operation to record the placements of the electrodes. The comparison between the MEG sources and the intracranial recordings was done at the gyrus level. The outcome of patients who underwent surgical resection was obtained by chart review. The time of follow up or clinic visits ranged from one to three months.

\section{Data analysis}

MEG data were visually inspected by a pediatric neurologist for epileptic spikes and artifacts. At least two epochs of MEG data (2-minute recordings) without noticeable artifacts (see the inclusion criteria for details) were then selected for frequency analysis. The frequency features of the measured MEG data in sensor space were analyzed with accumulated spectrogram (Xiang et al. 2004, Xiao et al. 2006). Morlet continuous wavelet transform was used for transforming time-domain data to frequency-domain data. Since accumulated spectrogram was based on wavelet time-frequency decomposition, it is synonymous with wavelet time-frequency decomposition with a modification that focuses on frequency resolution. With a fixed sigma value (number of oscillation), wavelet is sensitive to frequency at low-frequency and sensitive to time at high- 
frequency. To overcome this weakness and focus on the frequency characteristics of spontaneous brain activities, we used two sigma values for time-frequency analysis: one small value for low-frequency signals and one large value for high-frequency signals. For neuromagnetic signals in the $0.5-100 \mathrm{~Hz}$ band, the sigma was set to 6; for signals in the 100-2 $000 \mathrm{~Hz}$ band, the sigma was set to 48 . In low-frequency signal analysis, one epoch of data $(120 \mathrm{sec})$ was divided into 20 time segments of data; each time segment of data was $6 \mathrm{sec}$ (24 000 data points). In high-frequency signal analysis, one epoch data $(120 \mathrm{sec})$ was divided into 1000 time segments of data; each time segment of data was $0.12 \mathrm{sec}$ (480 data points). One spectrogram was computed for each time segment of data for each sensor. Twenty spectrograms were computed for one epoch of data for each sensor for low-frequency signals; one thousand spectrograms were computed for one epoch of data for each sensor for high-frequency signals. One accumulated spectrogram was produced by adding all 20 or 1000 spectrograms together (accumulation) for one epoch of data for each sensor. In this procedure, the different spectrograms of individual time segments were mathematically summed together to a single new overall spectrogram. An accumulated spectrogram can reveal brain activity in a consistent frequency range at multiple time windows. It can be considered as a "collective result" for a two-minute recording. Since each patient had two epochs of MEG data, two accumulated spectrograms were computed for each patient. Since the absolute spectral power varied across patients, the present study normalized the spectral power in a range of 0-100 for each patient. To identify the frequency profile of the entire brain, an accumulated spectrogram of global field (global spectrogram) was calculated from all MEG sensors for one epoch of data. The global spectrogram was an averaged spectrogram for all the accumulated spectrograms of all MEG sensors. Since each sensor was positioned in a distinct location around the head, the global spectrogram can be considered to be a "spatial summation" for each epoch of data. The underlying mathematical principles have been described in previous reports (Rau et al. 2002). The neuromagnetic activity at each sensor was visualized with contour maps, which showed small spectrograms at the position of each MEG sensor.

The frequency features of virtual sensor data in source space were analyzed using accumulated spectrogram. The virtual sensors were placed at the center of magnetic sources, which were volumetrically localized using wavelet based beamformer (Oishi et al. 2006, Xiang et al. 2004, Xiao et al. 2006). The beamformer algorithm implemented in this method was improved for performance and accuracy. The detailed mathematic algorithms have been described in previous reports (Brookes et al. 2007, Dalal et al. 2006, Sekihara et al. 2002). One of the major features of the improved beamformer in this study was its ability to detect correlated sources. A 3D-head model was created with each subject's MR image. For the magnetic spatial filtering analysis, multiple local spheres were fitted to each subject's head model. A customer-designed program, MEG Processor, was used to estimate magnetic sources for our MEG data. The program automatically placed virtual sensors at the center of the magnetic sources in the brain and then computed the accumulated spectrogram (Xiang et al. 2004, Xiao et al. 2006). The whole brain was scanned with a $2.5 \mathrm{~mm}$ resolution for sources. Similar to the aforementioned time-frequency analysis, virtual sensor data were translated from the time-domain waveform to the frequency-domain spectrogram using Morlet continuous wavelet transform. Neuromagnetic signals identified in the virtual sensor data were considered to be where the virtual sensor was placed. The distances between the MEG source locations and the lesions identified on MRI were measured with Magnetic Source Locator (Xiang et al. 2004, Xiao et al. 2006), a software package for three dimensional integration of MEG and MRI. The software was able to determine the center of the source localization automatically. Therefore, we could measure the distance between the MEG source localization and the border of lesions quantitatively.

\section{Results}

According to our routine MEG system and environmental noise test, the accumulated spectrograms computed from MEG data without subject showed activities around $60 \mathrm{~Hz}$ and $1040-1050 \mathrm{~Hz}$. We considered the $60 \mathrm{~Hz}$ noise to be power-line noise. The $1040-1050 \mathrm{~Hz}$ noise was probably from the MEG system and environmental devices. Since signals above 1 040-1 050 were very weak, the present study focused on signals at $0.5-1000 \mathrm{~Hz}$. Neuromagnetic signals at $60 \mathrm{~Hz}$ and above $1000 \mathrm{~Hz}$ were not analyzed.

\section{Physical sensor data}

Visual inspection of the waveform found that 24 out of the 30 patients had clear epileptic spikes (24/30, 80\%). A typical MEG spike is shown in the waveforms in figure 1 (see figure 1, Spikes). In spectrograms, one of the outstanding neuromagnetic components in the lowfrequency band $(0.5-100 \mathrm{~Hz}$ ) was around $10 \mathrm{~Hz}$ (see figure 2, F10). This component was considered as alpha activity $(8-12 \mathrm{~Hz})$. Neuromagnetic signals in this frequency range were identified in 21 patients (21/30, $70 \%)$. Another neuromagnetic component was around 1-3 Hz (figure 2, F1-3). This component was a complex, which included delta $(0.5-3 \mathrm{~Hz})$ and theta $(4-7 \mathrm{~Hz})$ activities in 24 patients $(24 / 30,80 \%)$. Neuromagnetic activity around $25 \mathrm{~Hz}$ and $20 \mathrm{~Hz}$ were also found in 19 (19/30, $63 \%)$ and $22(22 / 30,73 \%)$ patients, respectively. In addition, we noted activity at $30-40 \mathrm{~Hz}$ and $80-90 \mathrm{~Hz}$, but those components varied across patients. figure 2 shows the main low-frequency components. 


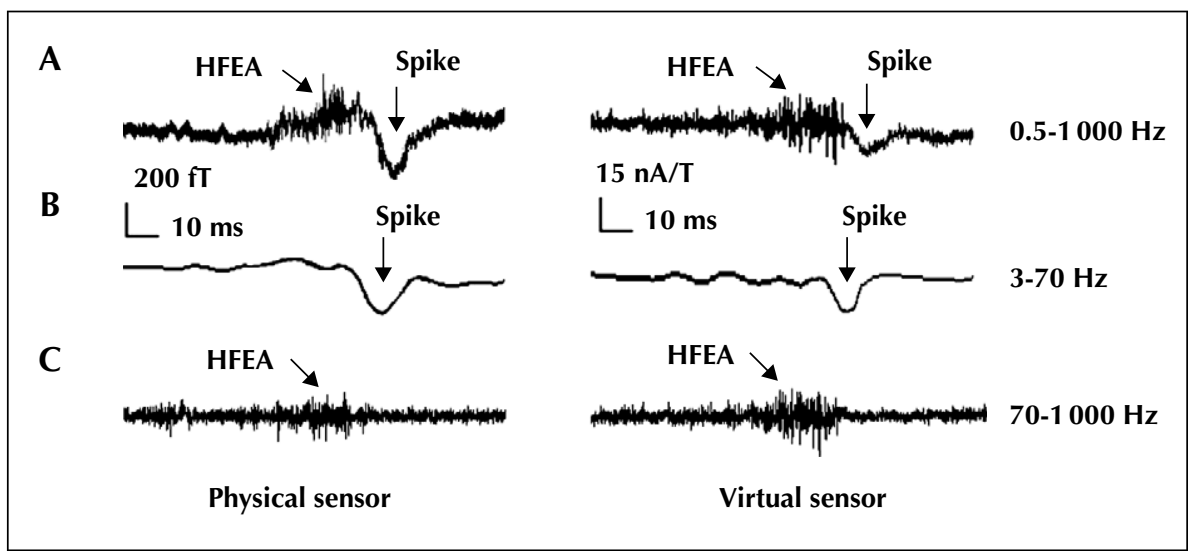

Figure 1. Waveforms of one MEG physical sensor and one virtual sensor data from a representative patient show high-frequency epileptic activity. The left column shows the waveforms of the physical sensor data ("Physical sensor"). The right column shows the waveforms of the virtual sensor data ("Virtual sensor"). The waveforms in row "A" are raw data at 0.5-1 $000 \mathrm{~Hz}$. The waveforms in row "B" are bandpass filtered with our routine setting $(3-70 \mathrm{~Hz})$ for detecting conventional spikes. The waveforms in row "C" are bandpass filtered with a new setting (70-1000 Hz) for detecting high-frequency epileptic activity ("HFEA"). The low- and high-frequency neuromagnetic signals are mixed in the raw waveform ("A"). The conventional spike can be clearly identified in the waveforms processed with a bandpass filter of 3-70 $\mathrm{Hz}$. Noticeably, the high-frequency epileptic activity is identifiable just before the conventional spike ("Spike").

The alpha activity (F10) was distributed in the occipital and temporal regions in 21 patients $(21 / 30,70 \%)$. The delta and theta (F1-3) activities were distributed in the left and right temporal and medial frontal regions in19 patients $(19 / 30,63 \%)$, and in the occipital region in five patients $(5 / 30,17 \%)$. Neuromagnetic activity above $20 \mathrm{~Hz}$ was distributed in focal regions and varied across patients. For example, the F20 was distributed in the right temporal region (see figure 2, F20).

Visual inspection of the waveforms found that 18 out of the 30 patients had very high-frequency activity (18/30, $60 \%$ ). A typical MEG high-frequency activity is shown in the waveforms in figure 1. Accumulated spectrograms (100-1 $000 \mathrm{~Hz}$ ) revealed at least one high-frequency component for 26 patients $(26 / 30,86 \%)$. The highest frequency was identified around $910 \mathrm{~Hz}$ (see figure 3, F910); the lowest frequency was identified around $110 \mathrm{~Hz}$ (see figure 3, F110). Noticeably, the exact frequency of high-frequency components varied across patients. The most consistent component was around $370 \mathrm{~Hz}$ (see figure 3, F370), which was found in 17 patients $(17 / 30,57 \%)$. We observed that 19 patients $(19 / 30,63 \%)$ had more than one high-frequency component. Of the 19 patients, seven patients had two highfrequency components and six patients had three highfrequency components. The main high-frequency components are shown in figure 3.

The distributions of high-frequency components (100$1000 \mathrm{~Hz}$ ) varied across patients. This observation implies that high-frequency components were probably pathogenic because the conventional physiogenic alpha activity (F10) was consistently distributed in the occipital regions. In the present study, we found high-frequency components in the temporal-frontal regions for 14 patients
$(13 / 30,43 \%)$, and in the occipital region for nine patients $(9 / 30,30 \%)$. The representative distributions of the highfrequency components are shown in figure 3.

In comparison to low-frequency neuromagnetic signals, the distributions of high-frequency components (100$1000 \mathrm{~Hz}$ ) were highly localized. We noted that the distributions of F20 and F25 in low-frequency components were similar to those of the high-frequency components in 11 patients $(11 / 30,36 \%)$. This observation implied that both F20/F25 and high-frequency components were probably epileptic activities.

Since we obtained two epochs of MEG data for each patient, we compared the two accumulated spectrograms for each frequency band. The comparison revealed that the two spectrograms showed the same results for 19 patients (64\%) and showed different results for 11 patients $(36 \%)$. For those patients who showed different components in the two accumulated spectrograms for either low- or high-frequency signals, we used only the frequency components that were identified in both of the two accumulated spectrograms.

\section{Virtual sensor data}

Visual inspection of virtual sensor data revealed that 24 out of the 30 patients had clear epileptic spikes (80\%). The outstanding frequency component in the spectrograms of virtual sensor data in low-frequency $(0.5-100 \mathrm{~Hz})$ was alpha activity in a range of $8-12 \mathrm{~Hz}$ (see figure 4, F10). This component was identified by a virtual sensor placed in the occipital region (see the Methods section for source estimation and virtual sensor placement). In this study, 22 patients showed this component $(22 / 30,73 \%)$. Neuromagnetic signals at 1-6 Hz (see 


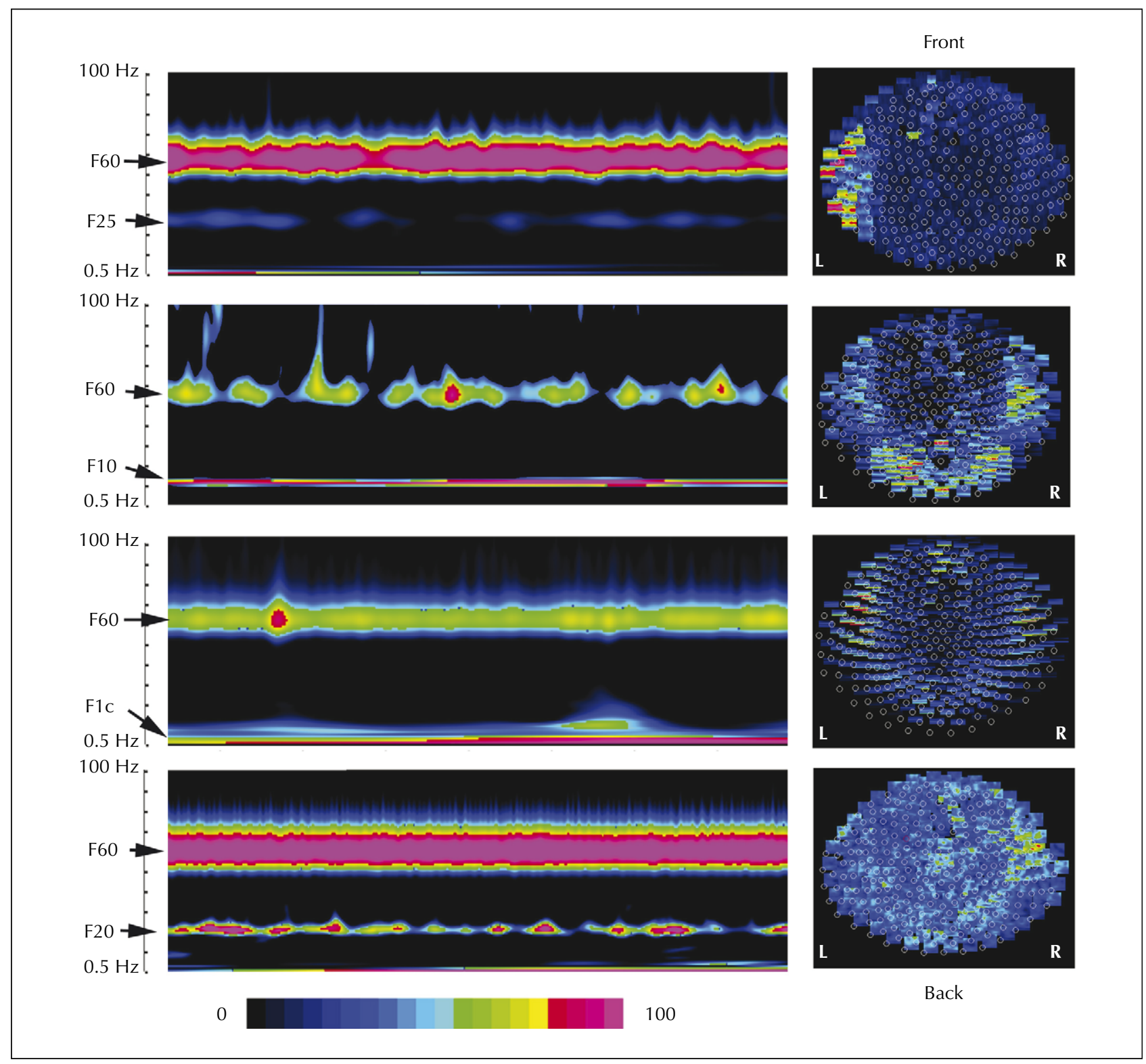

Figure 2. Four accumulated spectrograms of global fields and contour maps from four representative patients illustrate the main frequency components and distributions of neuromagnetic signals at $0.5-100 \mathrm{~Hz}$ in childhood epilepsy. The power-line noise (F60) is identifiable in all patients. Signals around 8-12 Hz (F10) are identified as alpha activity and are distributed around the occipital region. Signals around 0.5-7 Hz (F1c) are intermingled with activity around $0.5-3 \mathrm{~Hz}$ (delta) and 4-7 Hz (theta) and are mainly distributed in the left and right temporal regions. The activities around $20 \mathrm{~Hz}$ (F20) and $25 \mathrm{~Hz}$ (F25) show "periodic" patterns, which are probably caused by the "rhythmic burst" nature of the activity. The distributions of F20 and F25 are much more focal than that of F10 and F1c. All the spectrograms (on the left side) are in a range of $0.5-100 \mathrm{~Hz}$. In the contour map (on the right side), " $\mathrm{L}$ " indicates the left side of the head and " $\mathrm{R}$ " indicates the right side of the head. "Front" indicates the frontal region of the head at the upper part of the contour map; "Back" indicates the posterior region of the head at the lower part of the contour map. This orientation is the same for all contour maps. In the contour maps (on the right), each small circle represents one physical sensor. In the spectrograms (on the left), the $y$-axes indicate frequency ranges. Since multiple epochs of MEG data are accumulated, the interpretation of time on the x-axis is different to that of the conventional spectrogram. In accumulated spectrograms, the $\mathrm{x}$-axis indicates the width of time window without real time information; the $\mathrm{x}$-axis is therefore not labeled. The color bar shows the range of spectral power, which is normalized for each patient.

figure 4, F3c) were found for 25 patients $(25 / 30,83 \%)$ by a virtual sensor placed in the temporal region. Figure 4 shows the typical patterns of components in a frequency range of $0.5-100 \mathrm{~Hz}$ with virtual sensor technology. Neuromagnetic signals above $20 \mathrm{~Hz}$ (see figure 4, F22) were considered as epileptic activities and were found 


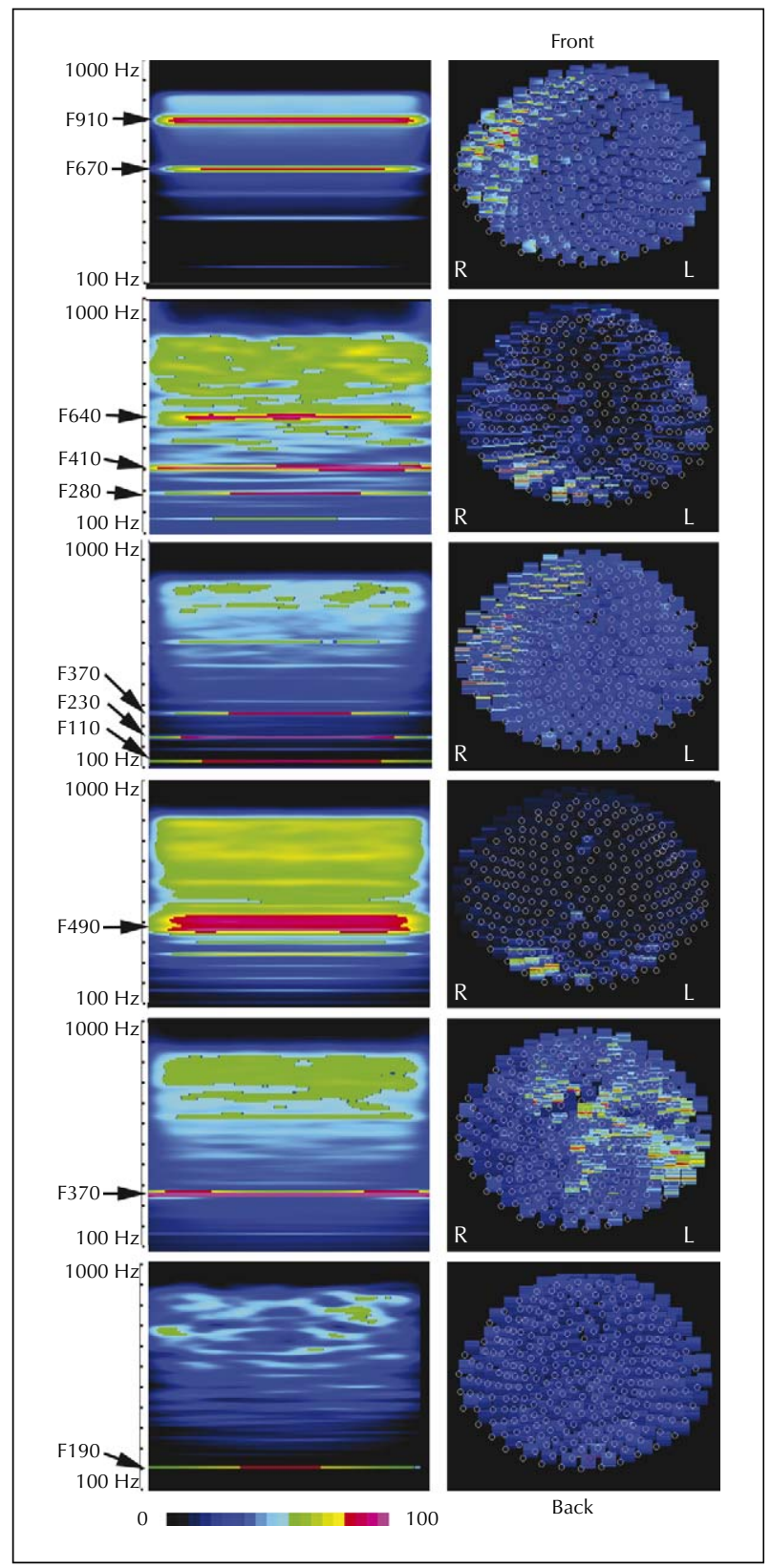

Figure 3. Six accumulated spectrograms of global fields and contour maps from six representative patients illustrate the main frequency components and distributions of neuromagnetic signals at $100-1000 \mathrm{~Hz}$ in childhood epilepsy. Noticeably, the activity patterns vary across patients. The highest frequency is around $910 \mathrm{~Hz}$ (F910) and is distributed over the left frontal-temporal region. The lowest frequency is around $110 \mathrm{~Hz}$ (F110) and is mainly distributed in the right frontal-temporal regions. Interestingly, multiple frequency components are identifiable in one patient. All the spectrograms (on the left side) are in a range of 100-1 $000 \mathrm{~Hz}$. In the contour map (on the right side), "L" indi-

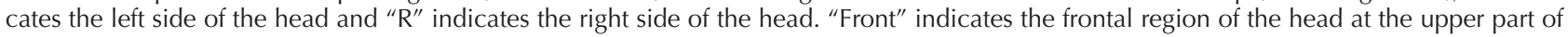
the contour map; "Back" indicates the posterior region of the head at the lower part of the contour map. This orientation is the same for all contour maps. In the contour maps (on the right), each small circle represents one physical sensor. In the spectrograms (on the left), the $y$ axes indicate frequency ranges. Since multiple epochs of MEG data are accumulated, the interpretation of time on the x-axis is different to that of the conventional spectrogram. In accumulated spectrograms, the x-axis indicates the width of time window without real time information; the $\mathrm{x}$-axis is therefore not labeled. The color bar shows the range of spectral power (0-100) which is normalized for each patient.

for 21 patients $(21 / 30,70 \%)$. The locations of the virtual sensors for those epileptic activities were in the vicinity of the lesions identified by MRI. The distances between the loci of the high-frequency epileptic activity and the lesions identified by MRI were measured for all of the 21 patients. The mean distance was $5.8 \pm 3.4 \mathrm{~mm}$. 


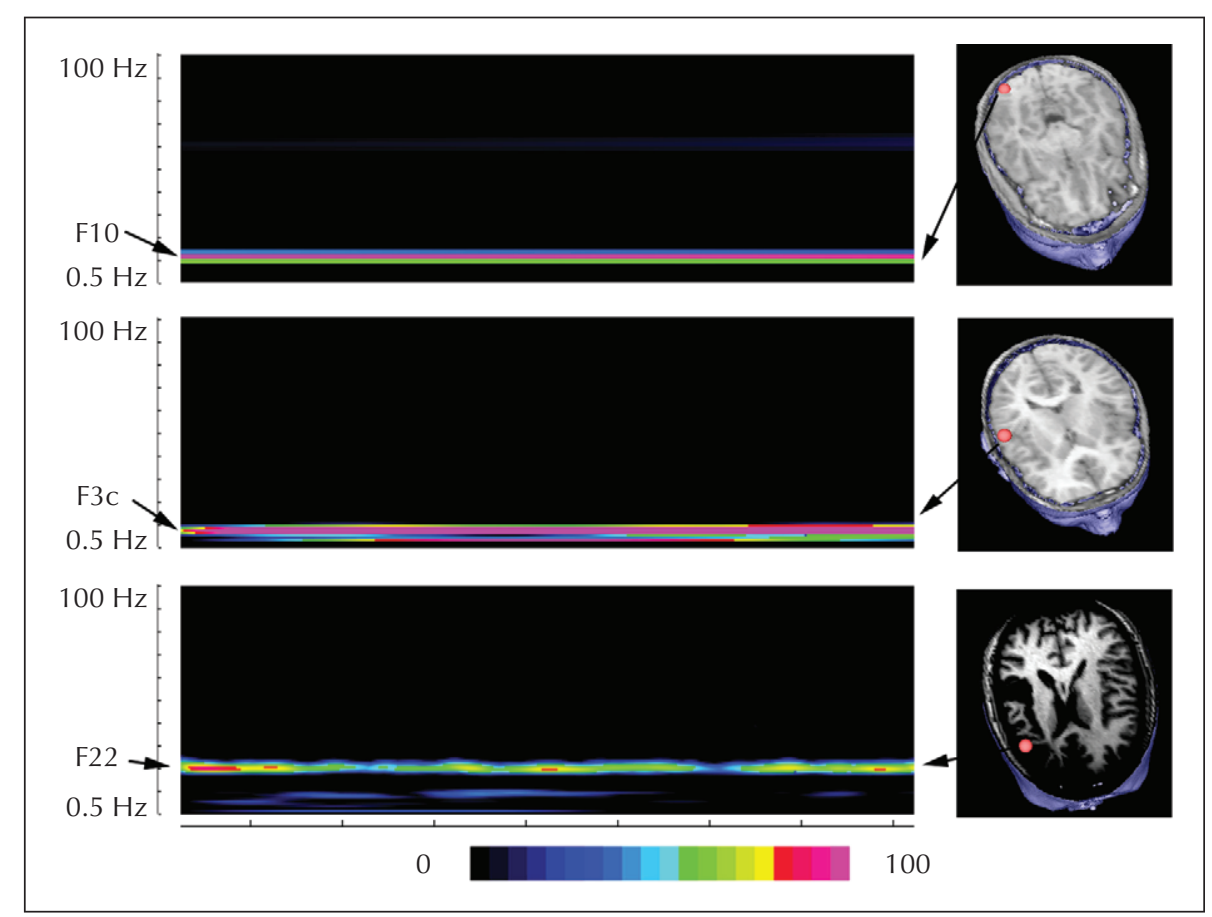

Figure 4. Three accumulated spectrograms of virtual sensor data from three representative patients illustrate the main frequency components at $0.5-100 \mathrm{~Hz}$ in childhood epilepsy. Signals around 8-12 Hz (F10) are identified as alpha activity and are found in the occipital cortices. Signals around $1-6 \mathrm{~Hz}(\mathrm{F3c})$ are intermingled with activity around $0.5-3 \mathrm{~Hz}$ (delta) and $4-7 \mathrm{~Hz}$ (theta) and are found in the right temporal cortex. The activity around $20-25 \mathrm{~Hz}$ is on the border of a lesion. All the spectrograms (on the left side) are in a range of $0.5-100 \mathrm{~Hz}$. In the contour maps (on the right), the small red circles represent the loci of the virtual sensors. In the spectrograms (on the left), the $y$-axes indicate frequency ranges. Since multiple epochs of MEG data are accumulated, the interpretation of time on the x-axis is different to that of the conventional spectrogram. In accumulated spectrograms, the $\mathrm{x}$-axis indicates the width of time window without real time information; the $\mathrm{x}$-axis is therefore not labeled. The color bar shows the range of spectral power which is normalized for each patient.

High-frequency spectrograms $(100-1000 \mathrm{~Hz})$ of virtual sensor data revealed high-frequency components for 26 patients $(26 / 30,86 \%)$. Of the 26 patients: one highfrequency component was found for seven patients, two components were found for seven patients, three components were found for six patients and four components were found for three patients. The highest frequency was identified around $910 \mathrm{~Hz}$ (see figure 5, F910); the lowest frequency was identified around $110 \mathrm{~Hz}$ (see figure 5, F110). Noticeably, high-frequency components varied across patients. The most consistent component was around $370 \mathrm{~Hz}$ (see figure 1, F370), which was found in 12 patients $(12 / 30,40 \%)$. Virtual sensors were found around the border of lesions in 21 patients $(21 / 30,70 \%)$. All high-frequency components were localized to the same epileptogenic lesions in 11 of the 19 patients who displayed multiple high-frequency components $(11 / 19,58 \%)$. Although the high-frequency components were localized in different brain areas of the remaining eight patients, the ultra highfrequency components (> $250 \mathrm{~Hz}$, or very fast ripple) were always localized to the epileptogenic lesions (8/8, 100\%). Therefore, the ultra high-frequency components were considered as epileptogenic activities. The main components of high-frequency epileptic activities are shown in figure 5. The distances between the loci of the high-frequency epileptic activities and the lesions identified by MRI were measured for all 21 patients. The mean distance was $4.3 \pm 2.7 \mathrm{~mm}$.

Since we obtained two epochs of MEG data for each patient, we computed two accumulated spectrograms for virtual sensor data in each frequency band. We compared the two accumulated spectrograms for each patient and found that the two spectrograms showed same results for 24 patients $(80 \%)$ and showed different results for six patients $(20 \%)$. For those patients who showed different components in the two accumulated spectrograms in either low- or high-frequency signals, we only used the frequency components that were identified in the two accumulated spectrograms.

In comparison to physical sensor data, virtual sensor data showed much less noise (figures 3, 4). Although 19 patients showed very similar frequency components in both physical sensors and virtual sensors (19/30, $63 \%)$, at least seven patients showed different frequency components $(7 / 30,23 \%)$. In addition, the comparison of the two spectrograms for each patient demonstrated that the virtual sensor data were more consistent than the physical sensor data ( $80 \%$ vs $64 \%$ ). The results implied that virtual sensor could minimize magnetic noise. 


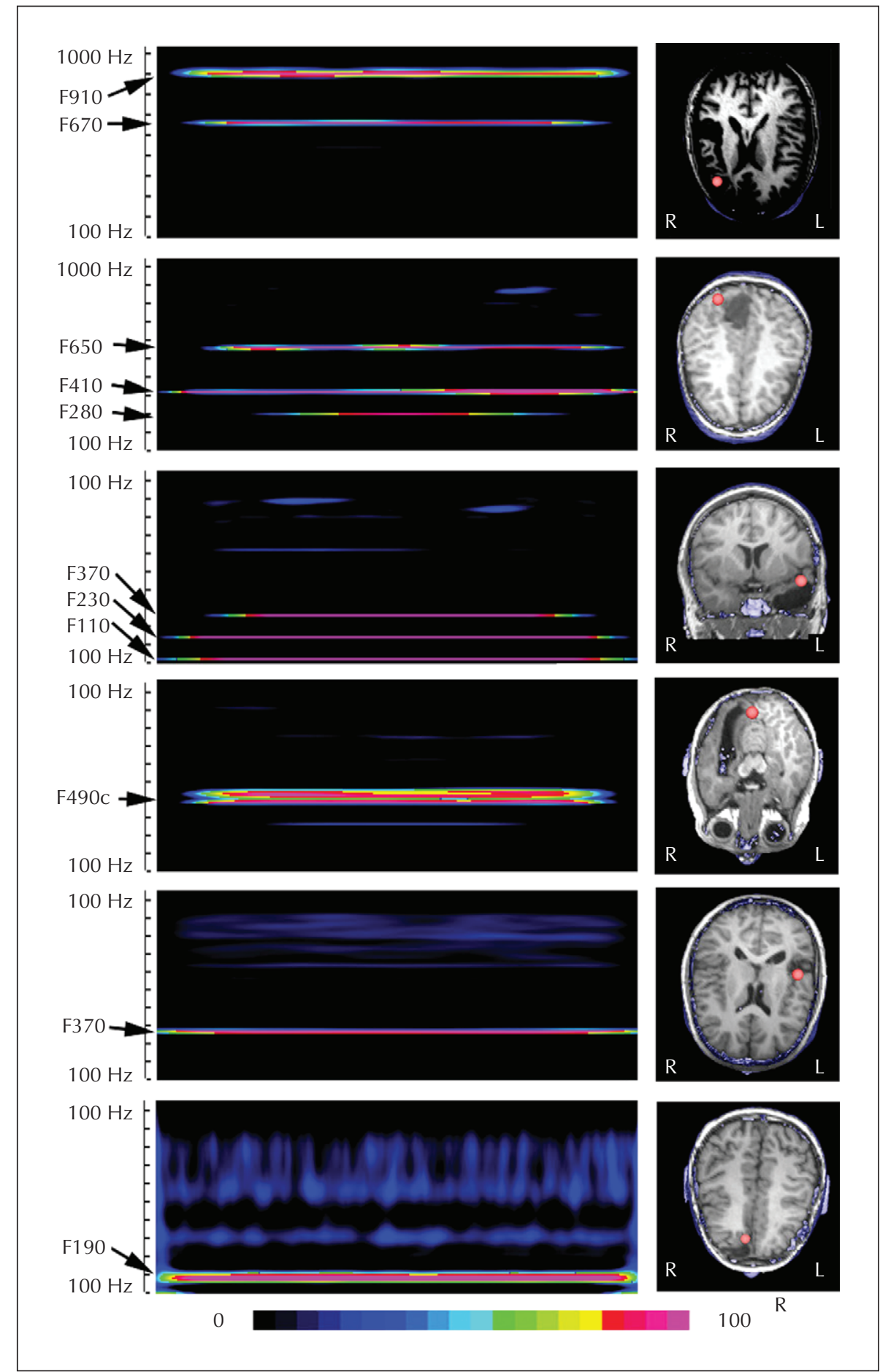

Figure 5. Six accumulated spectrograms of virtual sensor data from six representative patients illustrate the main frequency components at $100-1000 \mathrm{~Hz}$ in childhood epilepsy. The highest frequency is around $910 \mathrm{~Hz}$ (F910) and the loci of the virtual sensor are placed on the border of a lesion in the frontal cortex. The lowest frequency is around $110 \mathrm{~Hz}$ ( $\mathrm{F} 110$ ) and the virtual sensor is placed on the border of a lesion in the temporal cortex. Interestingly, multiple frequency components are identifiable in one patient. All the spectrograms (on the left side) are in a range of 100-1 $000 \mathrm{~Hz}$. In the contour maps (on the right), the small red circles represent the loci of the virtual sensors. In the spectrograms (on the left), the $y$-axes indicate frequency ranges. Since multiple epochs of MEG data are accumulated, the interpretation of the time on the $x$-axis is different to that of the conventional spectrogram. In accumulated spectrogram, the x-axis indicates the width of time window without real time information; the $\mathrm{x}$-axis is therefore not labeled. The color bar shows the range of spectral power which is normalized for each patient. 


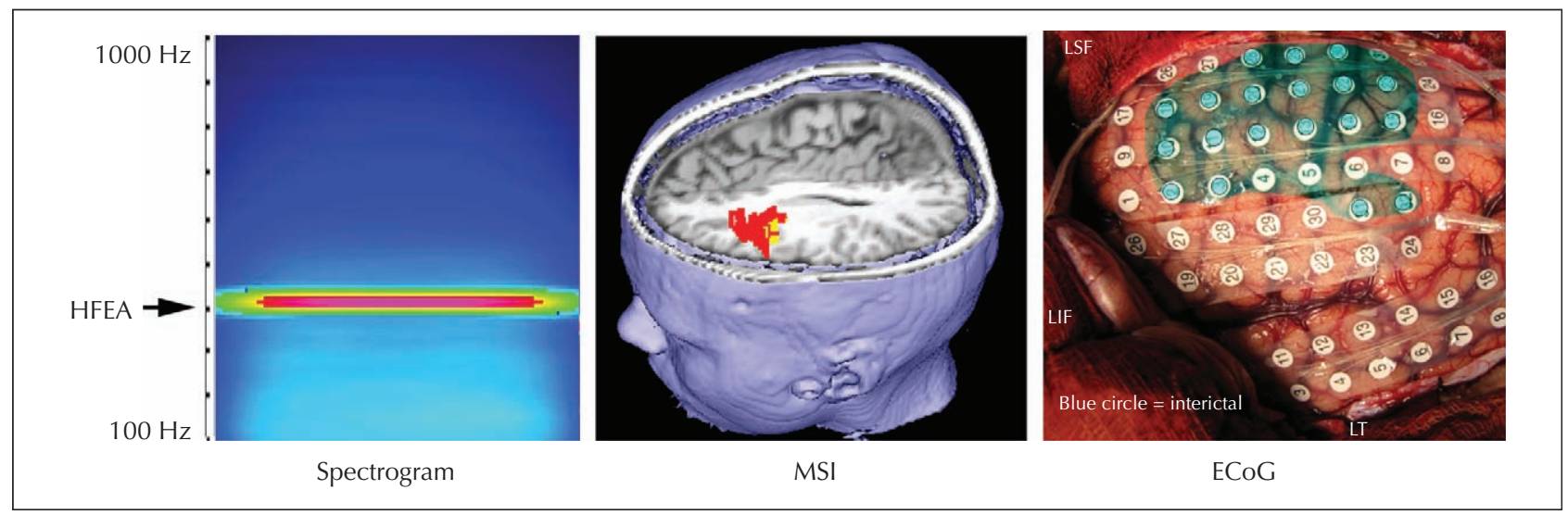

Figure 6. An accumulated spectrogram, magnetic source imaging ("MSI") and photograph of electrocorticography ("ECoG") from a representative patient show the concordance between MEG source localization of high-frequency epileptic activity ("HFEA") and ECoG (the "gold standard"). The accumulated spectrogram ("Spectrogram") reveals a high-frequency component around $410 \mathrm{~Hz}$ ("HFEA"; red line). The highfrequency component is volumetrically localized to the left medial frontal area ("MSI"; red and yellow region). Intracranial recording $($ "ECoG") shows the same region (blue area). In the ECoG photo, "LT" indicates the left temporal cortex and "LIF" indicates the left inferior frontal cortex. The MSI and ECoG noticeably localize to the same area.

\section{Correlations between MEG source localization and clinical data}

MEG high-frequency components correctly localized the epileptic areas for nine of the 12 patients who underwent clinical intracranial recordings $(9 / 12,75 \%)$. Of these, 11 patients had epilepsy surgery and one patient did not have a well-localized epileptic focus in the intracranial recording. The epileptic areas localized by MEG for two patients could not be validated by intracranial recordings. When the seizure onset zone determined by intracranial EEG was considered as the "gold standard" for localization of epileptic areas, $18 \%$ were false positive $(2 / 11)$. The sensitivity of MEG source localization at 100-1 $000 \mathrm{~Hz}$ was $82 \%(9 / 11)$. figure 6 shows an example of the magnetic source imaging of high-frequency epileptic activity and the intracranial recordings from a representative patient. The surgical outcomes were obtained by chart review. Of the 11 patients, eight patients had seizure freedom for at least one month after epilepsy surgery. The MEG source localization and intracranial recordings showed concordant results for all of the eight patients. Since the number of patients with true negative source localization was four, the specificity of MEG source localization at $100-1000 \mathrm{~Hz}$ was $67 \%(4 / 6)$.

\section{Discussion}

The results of the present study have demonstrated that high-frequency neuromagnetic signals in the range of $100-1000 \mathrm{~Hz}$ in pediatric patients can be noninvasively detected and volumetrically localized. We have been careful to eliminate high-frequency artifacts from our records, and it is unlikely that the main results reported here are due to measurement of artifacts. The reasons why we consider our data results from signals of the brain rather than noise can be summarized as follows. Firstly, we routinely conducted noise tests just before each clinical and research recording. We have identified that our system and environmental noise were around $60 \mathrm{~Hz}$ and 1 040-1 $050 \mathrm{~Hz}$. Secondly, the patient's head position was monitored with three coils. Our waveletbased beamformer could volumetrically localize the sources of neuromagnetic signals. If the signals were produced by noise, it should be localized to an area outside of the brain. Thirdly, similar to conventional averaging for detecting time-locked brain activity in evoked potentials, accumulated spectrogram detects frequency-locked brain activity. It has been documented that brain activity is commonly in a fixed frequency range (e.g. alpha activity is at $8-12 \mathrm{~Hz}$ ). However, magnetic noise commonly occurs at random frequency, with the exception of power-line noise. Furthermore, individual MRI was used as head model in this study. The combination of the accumulated spectrogram and virtual sensor techniques constrains the frequency of signals in the brain. In other words, our method mainly revealed the neuromagnetic signals in fixed frequency ranges in the brain, since the combination of the accumulated spectrogram and beamformer minimizes the signals which were not space- and frequency-locked. Therefore, we are confident that the high-frequency epileptic signals were truly brain signals, not noise. Our clinical data including intracranial recordings (the current "gold standard") and surgical outcomes have further validated our findings.

Previous studies of high-frequency electric signals in intracranial recording have been analyzed with bandpass filter and Fast Fourier transform (Bragin et al. 2004, Jirsch et al. 2006, Murakami et al. 2008, Urrestarazu et al. 2006, Worrell et al. 2008). The present study used accumulated 
spectrogram, a relatively new method for detecting high-frequency magnetic signals (Xiang et al. 2004). Accumulated spectrogram computes spectrograms from multiple epochs of data. The main reason for using accumulated spectrogram instead of single spectrogram in this study is that the neuromagnetic signals in the child's brain are very weak, and, according to our pilot study, it is very difficult to identify the brain signals in the frequency domain with single trial spectrogram. To remove environmental noise in random frequency range and obtain a better spectrogram, the present study used accumulated spectrogram to characterize the frequency profile in a wide frequency range for childhood epilepsy. Similar to conventional averaging for evoked potential, which relies on the fact that functional brain response is time-locked (the latency is nearly fixed), accumulated spectrogram relies on the fact that focal brain activity (e.g. alpha activity and epileptic activity) is space- and frequency-locked. Building on our previous report (Xiang et al. 2004), we have further improved the performance and accuracy of the existing accumulated spectrogram. Since accumulated spectrogram has clearly revealed alpha activity (8$12 \mathrm{~Hz}$ ) that has been widely recognized and confirmed for children and adults, we consider that accumulated spectrogram is a robust and suitable method for the present study. It should be noted that temporal information was not analyzed in the present study because epileptic activity is currently not predictable. It should also be noted that excellent time resolution could be obtained by changing the sigma value. However, to precisely determine the time and frequency, the use of multiple sigma values would be necessary, which goes beyond the scope of the present study.

High-frequency signals in MEG raw data include brain activity and magnetic noise. It has been reported that beamformer, or spatial filtering, can significantly minimize magnetic noise (Brookes et al. 2007, Dalal et al. 2006, Oishi et al. 2006, Sekihara et al. 2002). To minimize magnetic noise, we have further improved our method (Xiang et al. 2004) by integrating a virtual sensor technique with accumulated spectral analysis. Thus, we consider that the high-frequency signals detected by virtual sensor data indicate that the high-frequency signals in our results were from the brain. Although further investigation and confirmation are necessary, we consider that the determination of frequency components is reliable because the spectrograms were so clean (figures 3, 4). Additionally, one of the major improvements of beamformer in the present study is the ability to detect correlated sources. Building on our previous results (Oishi et al. 2006, Xiang et al. 2004, Xiao et al. 2006), the new beamformer algorithm can scan the entire brain and volumetrically estimate magnetic sources without making assumptions about the sources. The detailed mathematic algorithms have been well discussed in many previous publications (Brookes et al. 2007, Dalal et al. 2006,
Sekihara et al. 2002). We noted that alpha activity in the accumulated spectrogram from virtual sensor data was much clearer than that in the raw data. In addition, the $60 \mathrm{~Hz}$ noise was nearly invisible in the virtual sensor data. Since alpha activity and power-line noise $(60 \mathrm{~Hz}$ in our study) are classical EEG hallmarks, a clear alpha activity without power-line noise in our results suggests that the combination of accumulated spectrogram and beamformer can reliably detect brain signals even in a noisy environment.

One of the important findings of the present study is highfrequency neuromagnetic signals in childhood epilepsy. Previous reports (Bragin et al. 1999a, Jacobs et al. 2008, Jirsch et al. 2006, Urrestarazu et al. 2007, Worrell et al. 2004) on adult epilepsy with intracranial recordings have found that electric epileptic activity can reach $500 \mathrm{~Hz}$. The present results showed that high-frequency neuromagnetic signals in childhood epilepsy could reach $910 \mathrm{~Hz}$. The highest frequency in our MEG data is higher than that of the previous intracranial data in the human brain (Bragin et al. 2002, Bragin et al. 2004, Jacobs et al. 2008, Jirsch et al. 2006, Otsubo et al. 2008, Urrestarazu et al. 2006), but lower than that of animal model recording, which reaches $1500 \mathrm{~Hz}$ (Okada et al. 2005). Although the exact ceiling frequency needs further investigation and confirmation, according to our data and aforementioned previous reports, in children, the epileptic brain does generate high-frequency signals in a wide frequency range. Since the present data indicate that those signals are detectable non-invasively, we anticipate that the results of the present study will help us to further explore the clinical usefulness of high-frequency neuromagnetic signals in childhood epilepsy.

High-frequency neuromagnetic signals in childhood epilepsy can be divided into two categories. First, highfrequency neuromagnetic signals may be generated by functional activation, or physiogenic activity (Le Van Quyen et al. 2006). Second, high-frequency neuromagnetic signals can also be generated by epileptic activity, or pathogenic activity. To distinguish epileptic highfrequency signals from physiogenic signals, we overlapped virtual sensor with MRI. We noted that alpha activity was in the occipital cortex, while high-frequency neuromagnetic signals were around lesions for 22 and 21 patients, respectively. This observation suggests that the high-frequency components in the present study were probably generated by epileptogenic tissues around the lesions. Our results from intracranial recording strongly support our MEG findings. Furthermore, the structural images in the present study strongly support our MEG findings and recent publications (Guggisberg et al. 2008, Jacobs et al. 2008, Jirsch et al. 2006, Rampp and Stefan 2006, Urrestarazu et al. 2006) also support our results. Since the reliability of virtual sensor has been confirmed by intracranial recordings in a previous report (Oishi et al. 2002), we consider the high-frequency neuromagnetic 
signals around lesions to be pathogenic. Thus, we consider our results to be in line with recent reports. Taken together, our data suggest that high-frequency epileptic activity is highly localized in the seizure onset zone and is thought to have a causal role in the initiation of seizures or in epileptogenesis (Alegre et al. 2006, Della Marca et al. 2007, Jirsch et al. 2006, Worrell et al. 2008).

The cerebral mechanisms of high-frequency epileptic activity are not clear (Le Van et al. 2006, Murakami et al. 2008, Staba et al. 2004, Worrell et al. 2008). A recent report has suggested that high-frequency brain signals can be driven by GABA-mediated depolarization of interneuron networks in epileptic conditions and not physiologically at an earlier stage. These signals would trigger the cascades involved in epileptogenesis in the developing hippocampus (Le Van et al. 2006). Recurrent excitatory synaptic transmission and pyramidal axoaxonic gap junctions might thus be important for the very fast brain signals observed at seizure onset in the adult brain (Jirsch et al. 2006, Murakami et al. 2008, Urrestarazu et al. 2006, Worrell et al. 2008). From our point of view, MEG measures signals from a large group of neurons. High-frequency neuromagnetic signals could be produced by "out of phase" activities (Staley 2007). For example, if one group of neurons started to fire at the exact same time, one peak would be generated. However, if one group of neurons was divided into two subgroups which did not fire at the exact same time, two peaks would be generated. In a fixed time window, two peaks would basically double the frequency in measured data. Thus, the frequency of neuromagnetic signals from the brain may not solely depend on the frequency of neuron firing. Instead, high-frequency neuromagnetic signals in the epileptic brain may indicate "out of phase neural activities" or disorganized activities.

Another important finding of the present study is that highfrequency neuromagnetic signals in childhood epilepsy can be non-invasively recorded and volumetrically localized. It has been found that the frequencies of electric interictal high-frequency activity are well above the Berger EEG frequency bands $(0.1-30 \mathrm{~Hz})$, and are of such brief duration and low amplitude that by utilizing common clinical EEG viewing parameters (e.g. $10 \mathrm{~s}$ page and 0.5 $\mathrm{mV} / \mathrm{mm})$, these oscillations are obscured by lower frequency range activity. In fact, even when occurring as a sustained discharge at the seizure onset, the highfrequency activity is not always clearly apparent. The present study has shown that our new method can detect and visualize high-frequency brain activity in childhood epilepsy clearly (figures 2,4 ). We consider this result to be very important for the following reasons: (1) intracranial recordings are limited by their invasive nature; MEG is a non-invasive detection method and poses no risk to patients, (2) the brain area covered by intracranial recordings is usually limited to a small region. However, modern MEG, such as the MEG system used in the present study, can cover the entire brain. Although it is still debatable whether MEG can detect signals from the deep area of the brain, our experience indicates that MEG can at least provide clues and guidance for the electrode placement of intracranial recordings. We consider that the study of highfrequency epileptic activity may open a new window for clinical management of epilepsy. For example, if we are unable to capture conventional spikes for diagnosis of some patients with seizure(s), we might be able to capture high-frequency epileptic activities to confirm the diagnosis. Thus, the combination of accumulated spectrogram and virtual sensor technique may significantly change the way epilepsy is currently diagnosed. At least, volumetric localization of high-frequency epileptic activity could make a direct and important impact on epilepsy surgery. In summary, the results of the present study have demonstrated that childhood epilepsy is associated with highfrequency neuromagnetic signals in a wide frequency range. The highest frequency band was detected at $910 \mathrm{~Hz}$ and is far beyond the conventional epileptic spikes $(<70 \mathrm{~Hz})$. The correlations between MEG findings and clinical data suggest that the study of high-frequency epileptic activity may open a new window for clinical management of epilepsy. The measurement of high-frequency neuromagnetic signals in childhood epilepsy may also enable us to investigate the development of epilepsy noninvasively. The study of high-frequency brain activity in the developing brain may lead to a new understanding of the cerebral mechanisms underlying epileptogenesis.

\section{Disclosures.}

This study was supported by a Trustee Grant (120 000 dollars over two years) from Cincinnati Children's Hospital Medical Center, Cincinnati, $\mathrm{OH}$, USA. Dr Jing Xiang was the principal investigator (PI) of the research project.

\section{References}

Alegre M, Urriza J, Valencia M, Muruzábal J, Iriarte J, Artieda J. High-frequency oscillations in the somatosensory evoked potentials of patients with cortical myoclonus: pathophysiologic implications. J Clin Neurophysiol 2006; 23: 265-72.

Barkley GL, Baumgartner C. MEG and EEG in epilepsy. J Clin Neurophysiol 2003; 20: 163-78.

Bragin A, Engel J Jr, Wilson CL, Fried I, Buzsáki G. Highfrequency oscillations in human brain. Hippocampus 1999a; 9: 137-42.

Bragin A, Engel J Jr, Wilson CL, Fried I, Mathern GW. Hippocampal and entorhinal cortex high-frequency oscillations $(100--500 \mathrm{~Hz})$ in human epileptic brain and in kainic acidtreated rats with chronic seizures. Epilepsia 1999b; 40: 127-37.

Bragin A, Wilson CL, Almajano J, Mody I, Engel Jr. J. Highfrequency oscillations after status epilepticus: epileptogenesis and seizure genesis. Epilepsia 2004; 45: 1017-23.

Bragin A, Wilson CL, Staba RJ, Reddick M, Fried I, Engel Jr. J. Interictal high-frequency oscillations $(80-500 \mathrm{~Hz})$ in the human epileptic brain: entorhinal cortex. Ann Neurol 2002; 52: 407-15. 
Brookes MJ, Stevenson CM, Barnes GR, et al. Beamformer reconstruction of correlated sources using a modified source model. Neuroimage 2007; 34: 1454-65.

Dalal SS, Guggisberg AG, Edwards E, et al. Five-dimensional neuroimaging: localization of the time-frequency dynamics of cortical activity. Neuroimage 2008; 40: 1686-700.

Dalal SS, Sekihara K, Nagarajan SS. Modified beamformers for coherent source region suppression. IEEE Trans Biomed Eng 2006; 53: 1357-63.

Della Marca G, Vollono C, Barba C, Filomena Fuggetta M, Restuccia D, Colicchio G. High-frequency ECoG oscillations in the site of onset of epileptic seizures during sleep. Sleep Med 2007; 8: 96-7.

Draguhn A, Traub RD, Bibbig A, Schmitz D. Ripple (approximately $200-\mathrm{Hz}$ ) oscillations in temporal structures. JClin Neurophysiol 2000; 17: 361-76.

Firpi H, Smart O, Worrell G, Marsh E, Dlugos D, Litt B. Highfrequency oscillations detected in epileptic networks using swarmed neural-network features. Ann Biomed Eng 2007; 35: 1573-84.

Guggisberg AG, Kirsch HE, Mantle MM, Barbaro NM, Nagarajan SS. Fast oscillations associated with interictal spikes localize the epileptogenic zone in patients with partial epilepsy. Neuroimage 2008; 39: 661-8.

Iwasaki M, Pestana E, Burgess RC, Lüders HO, Shamoto $H$, Nakasato N. Detection of epileptiform activity by human interpreters: blinded comparison between electroencephalography and magnetoencephalography. Epilepsia 2005; 46: 59-68.

Jacobs J, Levan P, Chander R, Hall J, Dubeau F, Gotman J. Interictal high-frequency oscillations $(80-500 \mathrm{~Hz})$ are an indicator of seizure onset areas independent of spikes in the human epileptic brain. Epilepsia 2008; 49: 1893-907.

Jirsch JD, Urrestarazu E, Levan P, Olivier A, Dubeau F, Gotman J. High-frequency oscillations during human focal seizures. Brain 2006; 129: 1593-608.

Jung J, Mainy N, Kahane $\mathrm{P}$, et al. The neural bases of attentive reading. Hum Brain Mapp 2008; 29: 1193-206.

Le Van Quyen M, Khalilov I, Ben Ari Y. The dark side of highfrequency oscillations in the developing brain. Trends Neurosci 2006; 29: 419-27.

Murakami T, Sakuma K, Nomura T, Nakashima K, Hashimoto I. High-frequency oscillations change in parallel with short-interval intracortical inhibition after theta burst magnetic stimulation. Clin Neurophysiol 2008; 119: 301-8.

Oishi M, Kameyama S, Morota N, et al. Fusiform gyrus epilepsy: the use of ictal magnetoencephalography. Case report. J Neurosurg 2002; 97: 200-4.

Oishi M, Otsubo H, lida K, et al. Preoperative simulation of intracerebral epileptiform discharges: synthetic aperture magnetometry virtual sensor analysis of interictal magnetoencephalography data. J Neurosurg 2006; 105: 41-9.

Okada Y, Ikeda I, Zhang T, Wang Y. High-frequency signals $(>400 \mathrm{~Hz})$ : a new window in electrophysiological analysis of the somatosensory system. Clin EEG Neurosci 2005; 36: 285-92.
Otsubo H, Ochi A, Imai K, et al. High-frequency oscillations of ictal muscle activity and epileptogenic discharges on intracranial EEG in a temporal lobe epilepsy patient. Clin Neurophysiol 2008; 119: 862-8.

Raichle ME, MacLeod AM, Snyder AZ, Powers WJ, Gusnard DA, Shulman GL. A default mode of brain function. Proc Natl Acad Sci USA 2001; 98: 676-82.

Raichle ME, Snyder AZ. A default mode of brain function: a brief history of an evolving idea. Neuroimage 2007; 37: 1083-90.

Ramantani G, Boor R, Paetau R, et al. MEG versus EEG: influence of background activity on interictal spike detection. JClin Neurophysiol 2006; 23: 498-508.

Rampp S, Stefan H. Fast activity as a surrogate marker of epileptic network function? Clin Neurophysiol 2006; 117: 2111-7.

Rau R, Raschka C, Koch HJ. Uniform decrease of alpha-global field power induced by intermittent photic stimulation of healthy subjects. Braz J Med Biol Res 2002; 35: 605-11.

Salvador R, Martinez A, Pomarol-Clotet E, et al. A simple view of the brain through a frequency-specific functional connectivity measure. Neuroimage 2008; 39: 279-89.

Sekihara K, Nagarajan SS, Poeppel D, Marantz A. Performance of an MEG adaptive-beamformer technique in the presence of correlated neural activities: effects on signal intensity and timecourse estimates. IEEE Trans Biomed Eng 2002; 49: 1534-46.

Srinivasan R, Winter WR, Ding J, Nunez PL. EEG and MEG coherence: measures of functional connectivity at distinct spatial scales of neocortical dynamics. J Neurosci Methods 2007; 166: 41-52.

Staba RJ, Wilson CL, Bragin A, Jhung D, Fried I, Engel Jr. J. Highfrequency oscillations recorded in human medial temporal lobe during sleep. Ann Neurol 2004; 56: 108-15.

Staley KJ. Neurons skip a beat during fast ripples. Neuron 2007; 55: 828-30.

Urrestarazu E, Chander R, Dubeau F, Gotman J. Interictal highfrequency oscillations $(100-500 \mathrm{~Hz})$ in the intracerebral EEG of epileptic patients. Brain 2007; 130: 2354-66.

Urrestarazu E, Jirsch JD, Levan P, et al. High-frequency intracerebral EEG activity (100-500 Hz) following interictal spikes. Epilepsia 2006; 47: 1465-76.

Worrell GA, Gardner AB, Stead SM, et al. High-frequency oscillations in human temporal lobe: simultaneous microwire and clinical macroelectrode recordings. Brain 2008; 131: 928-37.

Worrell GA, Parish L, Cranstoun SD, Jonas R, Baltuch G, Litt B. High-frequency oscillations and seizure generation in neocortical epilepsy. Brain 2004; 127: 1496-506.

Xiang J, Holowka S, Qiao H, et al. Automatic localization of epileptic zones using magnetoencephalography. Neurol Clin Neurophysiol 2004: 98 (2004).

Xiao Z, Xiang J, Holowka S, et al. Volumetric localization of epileptic activities in tuberous sclerosis using synthetic aperture magnetometry. Pediatr Radiol 2006; 36: 16-21.

Ylinen A, Bragin A, Nadasdy Z, et al. Sharp wave-associated highfrequency oscillation $(200 \mathrm{~Hz})$ in the intact hippocampus: network and intracellular mechanisms. J Neurosci 1995; 15: 30-46. 